PROCEEDINGS OF THE

AMERICAN MATHEMATICAL SOCIETY

Volume 132, Number 4, Pages 1037-1047

S 0002-9939(03)07300-3

Article electronically published on November 7, 2003

\title{
ON PARTIAL ACTIONS AND GROUPOIDS
}

\author{
FERNANDO ABADIE
}

(Communicated by David R. Larson)

\begin{abstract}
We prove that, as in the case of global actions, any partial action gives rise to a groupoid provided with a Haar system, whose $C^{*}$-algebra agrees with the crossed product by the partial action.
\end{abstract}

\section{INTRODUCTION}

The theory of groupoid $C^{*}$-algebras has increasingly become a standard field of study in the area of operator algebras. Several large classes of $C^{*}$-algebras, such as AF-algebras, Cuntz and Cuntz-Krieger algebras, crossed products of commutative $C^{*}$-algebras, etc., may be expressed and studied as $C^{*}$-algebras associated with certain groupoids (general references on groupoid $C^{*}$-algebras are [16] and [13]). The aim of this paper is to add to the above list all crossed products of commutative $C^{*}$-algebras by partial actions.

The notion of crossed product by a partial action has its origin in the concept of crossed product by a partial automorphism introduced by Exel in [2]. Maclanahan defined in [11] partial actions and crossed products of $C^{*}$-algebras by partial actions of discrete groups, and Exel extended his definitions to the more general case of a twisted partial action of a locally compact group on a $C^{*}$-algebra. The reader is referred to [5] for the general definitions of partial actions and crossed products by partial actions. Since the introduction of partial automorphisms, several $C^{*}$ algebras have been described as crossed products of commutative $C^{*}$-algebras by partial actions: AF-algebras, Bunce-Deddens and Bunce-Deddens-Toeplitz algebras, Toeplitz algebras of quasi-lattice groups, etc. (see [4], [3], [8]). Partial actions have also been useful for generalizing Cuntz-Krieger algebras to the case of infinite matrices $([7])$. The construction carried out in this paper shows how all of the algebras mentioned above may be thought of as groupoid $C^{*}$-algebras.

In the case of a partial action $\theta$ of a discrete group $G$ on a space $X$, the groupoid $\mathcal{G}_{\theta}$ that we construct can be obtained by other means which we describe next. The proofs of our statements are routine, and we do not include them for lack of space. One possibility is to get $\mathcal{G}_{\theta}$ as the sheaf groupoid of germs of the semigroup generated by the partial action $\theta$, at least when $\theta$ is topologically free (see Remark 2.1). On the other hand, it is not hard to see that the semigroup $S(G)$ associated with $G$ according to $[6$ is an $F$-inverse semigroup, and that the groupoid associated

Received by the editors April 25, 2001 and, in revised form, October 24, 2002.

2000 Mathematics Subject Classification. Primary 46L05.

Key words and phrases. Groupoids, Fell bundles, partial actions.

This work was partially financied by Fapesp, Brazil, Processo No. 95/04097-9. 
with the corresponding action of $S(G)$ via Nica's theory ([12]) agrees with $\mathcal{G}_{\theta}$. Recall as well that Sieben ([17]) associates an inverse semigroup $S$ with every covariant representation $(\pi, u)$ of the system $\left(C_{0}(X), G, \theta\right)$. It can be shown that if $\pi \times u$ is faithful, then $S$ is an $\tilde{F}$-inverse semigroup, and that its corresponding Nica's groupoid is isomorphic to $\mathcal{G}_{\theta}$. In addition, the work of Nica in 12 can be related to that of Paterson on localizations ([13]). In fact, every action of an $\tilde{F}$-inverse semigroup gives rise to a localization (in the sense of [13]) in such a way that the groupoid $\mathcal{G}$ of this action is isomorphic to the Paterson's groupoid of the localization. In particular, the $C^{*}$-algebra of this groupoid is isomorphic to the crossed product of $C_{0}\left(\mathcal{G}^{0}\right)$ by the standard action of any full inverse semigroup of open $\mathcal{G}$-sets ([15]). Finally, we mention that another relation of the present paper with Paterson's work on groupoids is given by the fact that the universal groupoid of $S(G)$ is isomorphic to the groupoid of the partial action used to define the partial $C^{*}$-algebra of $G$.

The organization of this paper is the following. In the first section we define partial actions on topological spaces (see also [1]), and we show that our definition is the dual notion of partial actions on $C^{*}$-algebras. In Section 2 we extend the standard construction of the groupoid of a transformation group to the case of a partial action. The third section is devoted to proving that the crossed product by a partial action agrees with the $C^{*}$-algebra of the groupoid associated with it.

The author would like to express his gratitude to the referee for directing his attention to some work of which the author was unaware.

\section{Partial actions on $X$ and partial actions on $C_{0}(X)$}

In this section we show that the duality between the categories of locally compact spaces and commutative $C^{*}$-algebras provides a natural bijection between partial actions on $X$, defined below, and partial actions on $C_{0}(X)$.

Definition 1.1. A partial action of the topological group $G$ on the topological space $X$ is a pair $\theta=\left(\left\{X_{s}\right\}_{s \in G},\left\{\theta_{s}\right\}_{s \in G}\right)$ such that:

1. $X_{t}$ is open in $X$, and $\theta_{t}: X_{t^{-1}} \rightarrow X_{t}$ is a homeomorphism, $\forall t \in G$;

2. the set $D^{-1}:=\left\{(t, x) \in G \times X: t \in G, x \in X_{t^{-1}}\right\}$ is open in $G \times X$, and the map (also called $\theta$ ) $\theta: D^{-1} \rightarrow X$ given by $(t, x) \longmapsto \theta_{t}(x)$ is continuous;

3. $X_{e}=X$, and $\theta_{s t}$ is an extension of $\theta_{s} \theta_{t}, \forall s, t \in G$, where $e$ is the unit of $G$. If $\theta=\left(\left\{X_{t}\right\}_{t \in G},\left\{\theta_{t}\right\}_{t \in G}\right)$ and $\theta^{\prime}=\left(\left\{X_{t}^{\prime}\right\}_{t \in G},\left\{\theta_{t}^{\prime}\right\}_{t \in G}\right)$ are partial actions of $G$ on $X$ and $X^{\prime}$ respectively, we say that a continuous function $\phi: X \rightarrow X^{\prime}$ is a morphism $\phi: \theta \rightarrow \theta^{\prime}$ if $\phi\left(X_{t}\right) \subseteq X_{t}^{\prime}$, and $\phi \theta_{t}=\theta_{t}^{\prime} \phi, \forall t \in G$. If we forget the topological structures of $G$ and $X$, we say that $\theta$ is a set-theoretic partial action; note that in this case, condition 2 is superfluous, and condition 1 amounts to saying that every $\theta_{t}$ is a bijection.

Note that the restriction of a continuous action to an open subset is a partial action. Indeed, every partial action is of this type (see [1]). Another remarkable example of partial action is provided by the flow of a vector field.

Recall that a collection $\left\{E_{x}\right\}_{x \in X}$ of closed subspaces of a Banach space $E$, indexed by a topological space $X$, is called a continuous family, if for any open subset $U$ of $E$ the set $\left\{x \in X: U \cap E_{x} \neq \emptyset\right\}$ is open in $X$ (notion introduced in [5]).

Definition 1.2. Let $\sigma=\left(\left\{D_{t}\right\},\left\{\sigma_{t}\right\}\right)$ be a set-theoretic partial action of a locally compact Hausdorff group $G$ on a $C^{*}$-algebra $A$, where every $D_{t}$ is an ideal of $A$ 
and every $\sigma_{t}$ is an isomorphism of $C^{*}$-algebras. We say that $\sigma$ is a partial action if $\left\{D_{t}\right\}_{t \in G}$ is a continuous family and, if $\left(d_{t_{i}^{-1}}\right)_{i \in I}$ is a net in $A$ converging to $d_{t_{0}^{-1}} \in D_{t_{0}^{-1}}$, where $t_{i} \rightarrow t_{0}$ and $d_{t_{i}^{-1}} \in D_{t_{i}^{-1}}, \forall i \in I$, then $\sigma_{t_{i}}\left(d_{t_{i}^{-1}}\right) \rightarrow \sigma_{t_{0}}\left(d_{t_{0}^{-1}}\right)$. If $\sigma^{\prime}=\left(\left\{D_{t}^{\prime}\right\},\left\{\sigma_{t}^{\prime}\right\}\right)$ is a partial action of $G$ on $A^{\prime}$, then a morphism $\phi: \sigma \rightarrow \sigma^{\prime}$ is a homomorphism $\phi: A \rightarrow A^{\prime}$ such that $\phi\left(A_{t}\right) \subseteq A_{t}^{\prime}$ and $\phi \sigma_{t}=\sigma_{t}^{\prime} \phi, \forall t \in G$.

The restriction of a continuous action on a $C^{*}$-algebra to an ideal is a partial action, and every partial action on a $C^{*}$-algebra is obtained essentially in this way (see again [1]). In [5], Exel has shown that if $\sigma=\left(\left\{D_{t}\right\},\left\{\sigma_{t}\right\}\right)$ is a partial action of $G$ on the $C^{*}$-algebra $A$, then the bundle $\mathcal{B}:=\left\{(t, x): x \in D_{t}\right\} \subseteq G \times A$, with the relative topology, is a Fell bundle with product and involution given by (here $\left.x_{t} \delta_{t}:=\left(t, x_{t}\right) \in \mathcal{B}\right):\left(x_{t} \delta_{t}\right) *\left(x_{s} \delta_{s}\right)=\sigma_{t}\left(\sigma_{t}^{-1}\left(x_{t}\right) x_{s}\right) \delta_{t s}$ and $\left(x_{t} \delta_{t}\right)^{*}=\sigma_{t}^{-1}\left(x_{t}^{*}\right) \delta_{t^{-1}}$ respectively. This is the Fell bundle associated with $\sigma$. The cross-sectional algebra $C^{*}(\mathcal{B})$ of $\mathcal{B}$ is called the crossed product of $A$ by $\sigma$, and is denoted by $A \ltimes_{\sigma} G$ (for the theory of Fell bundles - also called $C^{*}$-algebraic bundles - we refer to [9]).

Let $X$ be a locally compact Hausdorff space, $G$ a locally compact Hausdorff group, and let $\theta=\left(\left\{\theta_{t}\right\}_{t \in G},\left\{X_{t}\right\}_{t \in G}\right)$ be such that $X_{t}$ is an open subset of $X$ and $\theta_{t}: X_{t^{-1}} \rightarrow X_{t}$ is a homeomorphism, $\forall t$. We will denote the family of such pairs $\theta$ by $\Theta$. Let $D=\left\{(t, x) \in G \times X: t \in G, x \in X_{t}\right\}, D^{-1}=\{(t, x) \in G \times X$ : $\left.t \in G, x \in X_{t^{-1}}\right\}$, and consider the map (also denoted by) $\theta: D^{-1} \rightarrow D$ such that $\theta(t, x)=\left(t, \theta_{t}(x)\right)$. We endow $D^{-1}$ and $D$ with the relative topologies inherited from the product topology on $G \times X$.

Consider now $A=C_{0}(X)$. We define the set $\Sigma$ to be the family of pairs $\sigma=\left(\left\{\sigma_{t}\right\}_{t \in G},\left\{D_{t}\right\}_{t \in G}\right)$, where $D_{t} \triangleleft A$ and $\sigma_{t}: D_{t^{-1}} \rightarrow D_{t}$ is a $C^{*}$-algebra isomorphism, $\forall t \in G$. Moreover, let $\mathcal{B}=\left\{(t, a) \in G \times A: t \in G, a \in D_{t}\right\}$, $\mathcal{B}^{-1}=\left\{(t, a) \in G \times A: t \in G, a \in D_{t^{-1}}\right\}$, and $\sigma: \mathcal{B}^{-1} \rightarrow \mathcal{B}$ be such that $\sigma(t, a)=\left(t, \sigma_{t}(a)\right)$. We also consider on $\mathcal{B}^{-1}$ and $\mathcal{B}$ the relative topologies inherited from the product topology on $G \times A$.

We have a bijection $\Phi: \Theta \rightarrow \Sigma$ : from $\theta$ we construct $\sigma=\left(\left\{\sigma_{t}\right\},\left\{D_{t}\right\}\right)$, where $D_{t}=C_{0}\left(X_{t}\right)$, considered as an ideal of $A$, and $\sigma_{t}: D_{t^{-1}} \rightarrow D_{t}$ is given by $\sigma_{t}(a)=$ $a \circ \theta_{t}^{-1}, \forall a \in D_{t}$. On the other hand, if $I$ and $J$ are ideals of $C_{0}(X)$, by Gelfand's theorem there exist unique open subsets $U, V \subseteq X$ such that $I=\{a \in A: a(x)=$ $0, \forall x \notin U\}$ and $J=\{a \in A: a(x)=0, \forall x \notin V\}$, and if $\sigma: I \rightarrow J$ is a $C^{*}$-algebra isomorphism, then there exists a unique homeomorphism $\theta: U \rightarrow V$ such that $\sigma(a)=a \circ \theta^{-1}$, that is, $\sigma(a)(x)=a\left(\theta^{-1}(x)\right)$ if $x \in V, \sigma(a)(x)=0$ otherwise.

Proposition 1.3. Let $\theta \in \Theta$ and $\sigma=\Phi(\theta) \in \Sigma$ be as above, and consider the following subspaces of $C_{0}(G, A): C_{0}(\mathcal{B})=\left\{f \in C_{0}(G, A): f(t) \in D_{t}, \forall t \in G\right\}$, and $C_{0}\left(\mathcal{B}^{-1}\right)=\left\{f \in C_{0}(G, A): f(t) \in D_{t^{-1}}, \forall t \in G\right\}$. Then

1. $C_{0}(\mathcal{B})$ and $C_{0}\left(\mathcal{B}^{-1}\right)$ are ideals of $C_{0}(G, A)$.

2. The following assertions are equivalent:

(a) $\left\{D_{t}\right\}_{t \in G}$ is a continuous family;

(b) $\mathcal{B}$ is a Banach bundle;

(c) for every $t \in G$ we have that $D_{t}=\left\{f(t): f \in C_{0}(\mathcal{B})\right\}$;

(d) the spectrum $\widehat{C_{0}(\mathcal{B})}$ of $C_{0}(\mathcal{B})$ agrees with $D$;

(e) $D$ is an open set.

Proof. It is clear that $C_{0}(\mathcal{B})$ and $C_{0}\left(\mathcal{B}^{-1}\right)$ are ideals of $C_{0}(G, A)$. The equivalence between $2(\mathrm{a}), 2(\mathrm{~b})$ and $2(\mathrm{c})$ follows from 3.2 and 3.3 of [5]. 
$(\mathrm{c}) \Longrightarrow(\mathrm{d})$ Since $C_{0}(\mathcal{B})$ is an ideal of $C_{0}(G, A) \cong C_{0}(G \times X), \widehat{C_{0}(\mathcal{B})}$ is the open subset of $G \times X$ formed by the points $(t, x) \in G \times X$ for which there exists $f \in C_{0}(\mathcal{B})$ such that $f(t)(x) \neq 0$. Pick an element $(t, x) \in D$. Then there exists $d \in D_{t}$ such that $d(x) \neq 0$. Since $\mathcal{B}$ is a Banach bundle over the locally compact space $G$, there exists a continuous section $F: G \rightarrow \mathcal{B}$ such that $F(t)=(t, d)$. Let $f: G \rightarrow A$ be such that $F(s)=(s, f(s))$. Then $f \in C_{0}(\mathcal{B})$ and $f(t)(x)=d(x) \neq 0$, whence $(t, x) \in \widehat{C_{0}(\mathcal{B})}$, and therefore $D \subseteq \widehat{C_{0}(\mathcal{B})}$. Conversely, if $(t, x) \notin D$, then $d(x)=0$, $\forall d \in D_{t}$, from which it follows that $f(t)(x)=0, \forall f \in C_{0}(\mathcal{B})$. Thus $\widehat{C_{0}(\mathcal{B})}=D$.

(d) $\Longrightarrow\left(\right.$ e) This is clear, because $\widehat{C_{0}(\mathcal{B})}$ is an open subset of $G \times X$.

(e) $\Longrightarrow$ (a) Let $a \in A, \epsilon>0$, and $U=\{x \in A:\|x-a\|<\epsilon\}$. We have to show that $G_{U}:=\left\{s \in G: U \cap D_{s} \neq \emptyset\right\}$ is open. We may suppose $G_{U} \neq \emptyset$. Let $t \in G_{U}$, and let $d \in U \cap D_{t}$. There is a compact set $K \subseteq X_{t}$ such that, $\forall y \notin K$ :

$$
|d(y)|<\epsilon-\|d-a\| .
$$

On the other hand, by the compactness of $K$ there exists a compact neighborhood $V$ of $t$, such that $V \times K \subseteq D$. Now, since $V \times K$ is a compact subset of the open set $D$, there exists a continuous function $g: G \times X \rightarrow[0,1]$, supported in $D$, and such that $g(s, y)=1$ if $(s, y) \in V \times K$. Now let $f: G \times X \rightarrow \mathbb{C}$ be such that $f(s, y)=d(y) g(s, y)$ and, for every $s \in G$, consider $d_{s}: X \rightarrow \mathbb{C}$ such that $d_{s}(y)=f(s, y)$. Since $\operatorname{supp}(g) \subseteq D$, we have that $\operatorname{supp}\left(d_{s}\right) \subseteq X_{s}, \forall s \in G$, and therefore $d_{s} \in D_{s}, \forall s \in G$. Now, if $s \in V$ :

$$
\left|d_{s}(y)-d(y)\right|=|d(y) g(s, y)-d(y)|=|d(y)|(1-g(s, y))<\epsilon-\|d-a\|,
$$

where the last inequality is due to the fact that if $y \in K$, then $g(s, y)=1$, while if $y \notin K$, then $d(x)<\epsilon-\|d-a\|$, by (10). It follows that:

$$
\left\|d_{s}-a\right\| \leq\left\|d_{s}-d\right\|+\|d-a\|<\epsilon .
$$

Thus, $d_{s} \in D_{s} \cap U, \forall s \in V$, and hence $t \in V \subseteq G_{U}$. So, $G_{U}$ is open.

Remark 1.4. Since the inversion on $G$ is a continuous map, it is clear that the family $\left\{D_{t}\right\}_{t \in G}$ is continuous if and only if the family $\left\{D_{t^{-1}}\right\}_{t \in G}$ is continuous, from which the equivalent assertions of 1.3 are also equivalent to the following ones:

(f) $\left\{D_{t^{-1}}\right\}_{t \in G}$ is a continuous family;

(g) $\mathcal{B}^{-1}$ is a Banach bundle;

(h) the spectrum $\widehat{C_{0}\left(\mathcal{B}^{-1}\right)}$ of $C_{0}\left(\mathcal{B}^{-1}\right)$ agrees with $D^{-1}$;

(i) $D^{-1}$ is an open set.

Proposition 1.5. Under the assumptions of Proposition 1.3 we have:

1. If $\left\{D_{t}\right\}_{t \in G}$ is a continuous family, the following assertions are equivalent:

(a) $\sigma: \mathcal{B}^{-1} \rightarrow \mathcal{B}$ is an isomorphism of Banach bundles;

(b) $\sigma: C_{0}\left(\mathcal{B}^{-1}\right) \rightarrow C_{0}(\mathcal{B})$ such that $\sigma(d)(t)=\sigma(d(t))$ is an isomorphism;

(c) $\theta: D^{-1} \rightarrow D$ is a homeomorphism.

2. $\sigma$ is a partial action on $A$ if and only if $\theta$ is a partial action on $X$.

Proof. Since $C_{0}(\mathcal{B})$ and $C_{0}\left(\mathcal{B}^{-1}\right)$ may be identified with the cross-sectional $C^{*}$ algebras of $\mathcal{B}$ and $\mathcal{B}^{-1}$ respectively, it is clear that $1(\mathrm{a})$ implies $1(\mathrm{~b})$. The converse implication follows from [9, II-13.16]. On the other hand, the equivalence between 1(b) and 1(c) is due to the fact that, by Proposition $1.3, D^{-1}$ and $D$ are the spectra of $C_{0}\left(\mathcal{B}^{-1}\right)$ and $C_{0}(\mathcal{B})$ respectively, and moreover $\sigma(a)=a \circ \theta^{-1}, \forall a \in C_{0}\left(\mathcal{B}^{-1}\right)$. 
As for 2, suppose that $\theta$ is a partial action on $X$. Recall that the map $U \mapsto C_{0}(U)$ is an order-preserving lattice isomorphism from the topology of $X$ to the family of ideals of $C_{0}(X)$, and therefore $D_{s} \cap D_{t}=C_{0}\left(X_{s} \cap X_{t}\right), \forall s, t \in G$. In particular, since $X_{t^{-1}} \cap X_{t^{-1} s^{-1}}$ is the domain of $\theta_{s} \theta_{t}$ and $\theta_{s} \theta_{t}\left(X_{t^{-1}} \cap X_{t^{-1} s^{-1}}\right)=X_{s} \cap X_{s t}$ (see 14, Lemma 1.2), it follows that $D_{t^{-1}} \cap D_{t^{-1} s^{-1}}$ is the domain of $\sigma_{t} \sigma_{s}$ and that $\sigma_{s} \sigma_{t}\left(D_{t^{-1}} \cap D_{t^{-1} s^{-1}}\right)=D_{s} \cap D_{s t}$. Now, if $d \in D_{t^{-1}} \cap D_{t^{-1} s^{-1}}$, and $x \in X_{s} \cap$ $X_{s t}$, then $\sigma_{s}\left(\sigma_{t}(d)\right)(x)=\sigma_{t}(d)\left(\theta_{s^{-1}}(x)\right)=d\left(\theta_{t^{-1}}\left(\theta_{s^{-1}}(x)\right)\right)=d\left(\theta_{t^{-1} s^{-1}}(x)\right)=$ $d\left(\theta_{(s t)^{-1}}(x)\right)=\sigma_{s t}(d)(x)$; if $x \in X_{s t} \backslash X_{s}, \theta_{(s t)^{-1}}(x) \in X_{(s t)^{-1}} \backslash X_{t^{-1}}$, then $\sigma_{s t}(d)(x)=0=\sigma_{s}\left(\sigma_{t}(d)\right)(x)$. Thus $\sigma_{s t}$ is an extension of $\sigma_{s} \sigma_{t}$. Moreover, $\sigma_{e}=i d_{A}$, and so $\sigma$ is a partial action. We leave to the reader the similar proof of the converse part.

\section{The GROUPOID OF A PARTIAL ACTION}

In this section we show that we can associate a locally compact groupoid with every partial action on a topological space. From now on we suppose that $G$ is a second countable locally compact Hausdorff group and that $X$ is a second countable locally compact Hausdorff space.

The shortest definition of groupoid is the following: a groupoid is a small category with inverses, that is, the class of objects of the category is actually a set, and every morphism is an isomorphism. The definition above is equivalent to the following one $([16, \mathrm{I}-1.1])$ : a groupoid $\mathcal{G}$ is a set with a product $(x, y) \mapsto x y$ defined on a subset $\mathcal{G}^{(2)} \subseteq \mathcal{G} \times \mathcal{G}$, called the set of composable pairs, and an inversion $x \mapsto x^{-1}$, which is a bijection in $\mathcal{G}$, and such that the following conditions are satisfied:

1. $\left(x^{-1}\right)^{-1}=x, \forall x \in \mathcal{G}$;

2. if $(x, y),(y, z) \in \mathcal{G}^{(2)}$, then $(x y, z),(x, y z) \in \mathcal{G}^{(2)}$, and $(x y) z=x(y z)$;

3. $\left(x^{-1}, x\right) \in \mathcal{G}^{(2)}, \forall x \in \mathcal{G}$, and if $(x, y) \in \mathcal{G}^{(2)}$, then $x^{-1}(x y)=y$;

4. $\left(x, x^{-1}\right) \in \mathcal{G}^{(2)}, \forall x \in \mathcal{G}$, and if $(z, x) \in \mathcal{G}^{(2)}$, then $(z x) x^{-1}=z$.

If $x \in \mathcal{G}$, the domain of $x$ is $x^{-1} x$, and its range is $x x^{-1}$. Thus we have two maps $d, r: \mathcal{G} \rightarrow \mathcal{G}$, with the same image, $\mathcal{G}^{(0)}$, which is called the unit space of $\mathcal{G}$. In the category setting, the unit space is nothing but the set of objects.

The groupoid $\mathcal{G}$ is said to be locally compact if it has a second countable locally compact Hausdorff topology on it, compatible with its algebraic structure, i.e.:

1. The inversion $\mathcal{G} \rightarrow \mathcal{G}$ is continuous.

2. The product $\mathcal{G}^{(2)} \rightarrow \mathcal{G}$ is continuous, where $\mathcal{G}^{(2)}$ is endowed with the topology inherited from the product topology on $\mathcal{G} \times \mathcal{G}$.

Let $\theta=\left(\left\{X_{t}\right\}_{t \in G},\left\{\theta_{t}\right\}_{t \in G}\right)$ be a partial action of $G$ on $X$, and define $A_{y}:=\{t \in$ $\left.G:(t, y) \in D^{-1}\right\}$. We associate with $\theta$ the following category $\mathcal{G}$ (or $\mathcal{G}_{\theta}$ if it is necessary to make a distinction). The class of objects of $\mathcal{G}$ is the set $X$. If $y, x \in X$, we set $\operatorname{Mor}(y, x):=\left\{(x, t, y): t \in A_{y}\right.$, and $\left.x=\theta_{t}(y)\right\}$, with the composition law: $\operatorname{Mor}(y, x) \times \operatorname{Mor}(z, y) \rightarrow \operatorname{Mor}(z, x):((x, r, y),(y, s, z)) \longmapsto(x, r s, z)$. Since $\theta_{r s}$ is an extension of $\theta_{r} \theta_{s}$, if $s \in A_{z}$ and $r \in A_{\theta_{s}(z)}$, then $r s \in A_{z}$, and so $(x, r s, z) \in \operatorname{Mor}(z, x)$. We have over $x \in X$ the identity morphism $(x, e, x)$, which may be identified with $x$. Every morphism $(x, t, y)$ is an isomorphism, with inverse $(x, t, y)^{-1}=\left(y, t^{-1}, x\right)$. On the other hand, the associativity of the composition law follows from the associativity of the multiplication in the group $G$. Thus $\mathcal{G}$ is a groupoid. 
We will consider $X$ included in $\mathcal{G}$ via the map $\iota: X \rightarrow \mathcal{G}$ such that $x \longmapsto(x, e, x)$. The domain and range maps $d: \mathcal{G} \rightarrow X$ and $r: \mathcal{G} \rightarrow X$ are given by $d(x, t, y)=y$ and $r(r, t, y)=x$. We have that $r \iota=i d_{X}=d \iota$.

Note that $\mathcal{G}$ is the graph of the partial action $\theta$, written in a nonconventional form: instead of writing $\theta(t, x)$ to the right of $(t, x)$, we are writing it to the left, and this is why $\mathcal{G}$ is included in $X \times G \times X$ instead of $G \times X \times X$. This way, the multiplication and inversion in $\mathcal{G}$ are easily visualized and computed.

Now, we consider on $\mathcal{G}$ the topology inherited from the product topology on $X \times G \times X$. Since $\mathcal{G}$ is the graph of the partial action $\theta$, then $\mathcal{G}$ is homeomorphic to $D^{-1}$. In particular, $\mathcal{G}$ is locally compact. Moreover, by the continuity of $\theta$ the operations of inversion and product on $\mathcal{G}$ are continuous; so $\mathcal{G}$ is a locally compact groupoid. Also note that every morphism $\phi: \theta \rightarrow \theta^{\prime}$ between partial actions gives rise to a continuous homomorphism $\mathcal{G}_{\theta} \rightarrow \mathcal{G}_{\theta^{\prime}}$ between the corresponding groupoids such that $(x, t, y) \mapsto(\phi(x), t, \phi(y))$. In other words, we have defined a functor from the category of partial actions to the category of groupoids.

We have homeomorphisms $p: \mathcal{G} \rightarrow D$ and $q: \mathcal{G} \rightarrow D^{-1}$, where $p(x, t, y)=(t, x)$, and $q(x, t, y)=(t, y)$. Indeed, in order to handle the $C^{*}$-algebra of the groupoid $\mathcal{G}$, to be defined later, it is better to identify $\mathcal{G}$ with $D$ by means of the map $p$. With this identification, the inverse of $(t, x) \in D$ is $(t, x)^{-1}=\left(t^{-1}, \theta_{t^{-1}}(x)\right)$ and, if $(r, x),(s, y) \in D$, then $((r, x),(s, y))$ is a composable pair if and only if $\theta_{r^{-1}}(x)=y$, and in this case we have $(r, x)(s, y)=(r s, x)$. Finally, $\iota, r$ and $d$ take the forms: $\iota(x)=(e, x), r(t, x)=x$, and $d(t, x)=\theta_{t^{-1}}(x)$. We will see next that the translation of the algebraic structure of $\mathcal{G}$ to the set $D$ allows us to define a natural Haar system on the groupoid.

Remark 2.1. Suppose that $G$ is discrete, and let $\mathrm{PHom}(X)$ be the inverse semigroup of partial homeomorphisms between open subsets of the space $X$. Suppose in addition that $\theta$ is topologically free, that is, if $t \neq e$, then the set $\left\{x: \theta_{t}(x)=x\right\}$ has empty interior. For $f \in \operatorname{PHom}(X), x \in \operatorname{domain}(f)$, denote by $[f, x]$ the germ of $(f, x)$. Let $S:=\left\{\theta_{t_{1}} \cdots \theta_{t_{k}}: k \geq 0, t_{1}, \ldots, t_{k} \in G\right\}$ be the inverse semigroup of PHom $(X)$ generated by the partial action $\theta$. The inverse subsemigroups of $\operatorname{PHom}(X)$ are usually called pseudogroups. Note that $U_{t_{1}, \ldots, t_{k}}:=\operatorname{domain}\left(\theta_{t_{1}} \cdots \theta_{t_{k}}\right)$ is an open subset of $X_{\left(t_{1} \cdots t_{k}\right)^{-1}}$, and $\theta_{t_{1} \cdots t_{k}}$ agrees with $\theta_{t_{1}} \cdots \theta_{t_{k}}$ on $U_{t_{1}, \ldots, t_{k}}$. Thus $\left[\theta_{t_{1}} \cdots \theta_{t_{k}}, x\right]=\left[\theta_{t_{1} \cdots t_{k}}, x\right]$, for every $x \in U_{t_{1}, \ldots, t_{k}}$. Therefore, the space $\mathcal{G}_{S}$ of germs $\left\{\left[\theta_{t_{1}} \cdots \theta_{t_{k}}, x\right]: x \in U_{t_{1}, \ldots, t_{k}}\right\}$ has a natural structure of locally compact groupoid (10, Exemple, p. 257]). This groupoid is known as the sheaf groupoid of germs of the pseudogroup $S$. It is readily checked that the map $\mathcal{G} \rightarrow \mathcal{G}_{S}$ given by $(y, t, x) \mapsto$ $\left[\theta_{t}, x\right]$ is an isomorphism of locally compact groupoids. For a general $\theta$ we have that $\mathcal{G}$ is isomorphic to $\tilde{\mathcal{G}}_{S}:=\left\{\left(t,\left[\theta_{t}, x\right]\right) \in G \times \mathcal{G}_{S}\right\}$ via the map $(t, x) \mapsto\left(t,\left[\theta_{t}, x\right]\right)$.

2.1. Haar system on $\mathcal{G}$. Our immediate task will be to find a left Haar system on $\mathcal{G}$ (identified with $D$ as above). Recall that a left Haar system on a locally compact groupoid $\mathcal{G}$ ([16, I-2.2]) is a family $\left\{\lambda^{x}: x \in \mathcal{G}^{(0)}\right\}$ of measures on $\mathcal{G}$, such that:

1. $\operatorname{supp}\left(\lambda^{x}\right)=\mathcal{G}^{x}:=\{\gamma \in \mathcal{G}: r(\gamma)=x\}$;

2. Continuity: if $f \in C_{c}(\mathcal{G})$, the map $\nu_{f}: \mathcal{G}^{(0)} \rightarrow \mathbb{C}$ such that $\nu_{f}(x)=$ $\int_{\mathcal{G}} f(\gamma) d \lambda^{x}(\gamma)$ is continuous;

3. Left invariance: for any $f \in C_{c}(\mathcal{G})$ and any $x \in \mathcal{G}^{(0)}: \int_{\mathcal{G}} f\left(\gamma_{1} \gamma_{2}\right) d \lambda^{d\left(\gamma_{1}\right)}\left(\gamma_{2}\right)=$ $\int_{\mathcal{G}} f\left(\gamma_{2}\right) d \lambda^{r\left(\gamma_{1}\right)}\left(\gamma_{2}\right)$. 
To define the Haar system we are looking for, let us consider, for $x \in X$, the measure $\lambda^{x}$ on the Borel sets of $\mathcal{G}$ defined by: $\lambda^{x}(B)=\lambda \times \delta_{x}(B)$, where $B$ is a Borel set, $\lambda$ is the left Haar measure on $G$, and $\delta_{x}$ is the Dirac measure concentrated in $x$. In other words, $\lambda^{x}$ is the restriction to $D$ of the product measure $\lambda \times \delta_{x}$. Note that, since $\mathcal{G}$ is open in $G \times X, B \subseteq \mathcal{G}$ is a Borel set in $\mathcal{G}$ if and only if it is a Borel set in $G \times X$. Also note that, since $\mathcal{G}$ is an open subset of $G \times X$, we may suppose that any $f \in C_{c}(\mathcal{G})$ is defined on all of $G \times X$, with $f(s, y)=0$ for $(s, y) \notin \mathcal{G}$.

Proposition 2.2. The family $\Lambda=\left\{\lambda^{x}: x \in X\right\}$ is a left Haar system on $\mathcal{G}$.

Proof. Let us see first that $\operatorname{supp}\left(\lambda^{x}\right)=\mathcal{G}^{x}$. A simple computation shows that $\mathcal{G}^{x}=A_{x}^{-1} \times\{x\}$. On the other hand, consider two nonempty open subsets $A$ and $B$ of $G$ and $X$ respectively, such that $A \times B \subseteq \mathcal{G}$ (recall that $\mathcal{G}$ is open in $G \times X$ ). We have $\lambda^{x}(A \times B)=\lambda(A) \delta_{x}(B)=\chi_{B}(x) \lambda(A)$, where $\chi_{S}$ denotes the characteristic function of the set $S$. Since $\lambda(A)>0$, it follows that $\lambda^{x}(A \times B) \neq 0$ if and only if $x \in B$. Therefore the support of $\lambda^{x}$ is equal to $\mathcal{G} \cap(G \times\{x\})=A_{x}^{-1} \times\{x\}$.

Let us check now the continuity property. Let $f \in C_{c}(\mathcal{G})$, and consider the map $\nu_{f}: X \rightarrow \mathbb{C}$ such that $\nu_{f}(x)=\int_{\mathcal{G}^{x}} f(\gamma) d \lambda^{x}(\gamma)$. Then:

$$
\nu_{f}(x)=\int_{\{x\}} \int_{A_{x}^{-1}} f(s, y) d \lambda(s) d \delta_{x}(y)=\int_{G} \chi_{A_{x}^{-1}}(s) f(s, x) d \lambda(s) .
$$

If $K=p_{1}(\operatorname{supp}(f))$, where $p_{1}: G \times X \rightarrow G$ is the projection on the first coordinate, then $\nu_{f}(x)=\int_{K} f(s, x) d \lambda(s)$. If $x \in X$, the function $f_{x}: K \rightarrow \mathbb{C}$ such that $f_{x}(s)=f(s, x)$ is continuous; thus, $\xi: X \rightarrow C(K)$ such that $\xi(x)=f_{x}$ is continuous as well. Moreover, the map $\lambda_{K}: C(K) \rightarrow \mathbb{C}$ such that $g \longmapsto \int_{K} g(s) \lambda(s)$ is a continuous linear functional. Since $\nu_{f}=\lambda_{K} \xi$, it follows that $\nu_{f}$ is continuous.

It remains to verify the left invariance, that is, to show that $\forall f \in C_{c}(\mathcal{G})$ and $\forall(t, x) \in \mathcal{G}$ we have $\int_{\mathcal{G}^{d(t, x)}} f((t, x)(s, y)) d \lambda^{d(t, x)}(s, y)=\int_{\mathcal{G}^{r(t, x)}} f(s, y) d \lambda^{r(t, x)}(s, y)$. Since $\mathcal{G}^{x}=A_{x}^{-1} \times\{x\}$, we have that $\int_{\mathcal{G}^{r(t, x)}} f(s, y) d \lambda^{r(t, x)}(s, y)=\int_{A_{x}^{-1}} f(s, x) d \lambda(s)$. Let $z=d(t, x)=\theta_{t^{-1}}(x)$, and put $J:=\int_{\mathcal{G}^{d(t, x)}} f((t, x)(s, y)) d \lambda^{d(t, x)}(s, y)$. Then

$$
J=\int_{A_{z}^{-1}} f(t s, x) d \lambda(s)=\int_{G} \chi_{A_{z}^{-1}}\left(t^{-1} s\right) f(s, x) d \lambda(s) .
$$

Now, $A_{z}=A_{x} t$; so $t^{-1} s \in A_{z}^{-1} \Longleftrightarrow s^{-1} t \in A_{x} t \Longleftrightarrow s^{-1} \in A_{x} \Longleftrightarrow s \in A_{x}^{-1}$. Thus $\chi_{A_{z}^{-1}}\left(t^{-1} s\right) \neq 0 \Longleftrightarrow \chi_{A_{x}^{-1}}(s) \neq 0$. Consequently,

$$
J=\int_{G} \chi_{A_{x}^{-1}}(s) f(s, x) d \lambda(s)=\int_{A_{x}^{-1}} f(s, x) d \lambda(s)=\int_{\mathcal{G}^{r(t, x)}} f(s, y) d \lambda^{r(t, x)}(s, y),
$$

and so $J=\int_{\mathcal{G}^{r(t, x)}} f(s, y) d \lambda^{r(t, x)}(s, y)$, which ends the proof.

\section{Crossed product and the groupoid $\mathrm{C}^{*}$-Algebra}

In this final section we prove the main result of the paper: if $\theta$ is a partial action of $G$ on $X$, with induced partial action $\sigma:=\Phi(\theta)$ on $C_{0}(X)$, then $C_{0}(X) \ltimes_{\sigma} G \cong C^{*}(\mathcal{G})$, where the latter is the groupoid $C^{*}$-algebra of $\mathcal{G}$, and $\mathcal{G}=\mathcal{G}_{\theta}([16])$. 
Following the theory developed in [16] for our groupoid $\mathcal{G}$, we have that $C_{c}(\mathcal{G})$ is a *algebra with the product and involution given respectively by

$$
\begin{gathered}
f \star g(t, x)=\int_{\mathcal{G}^{d(t, x)}} f((t, x)(s, y)) g\left((s, y)^{-1}\right) d \lambda^{d(t, x)}(s, y), \\
f^{\star}(t, x)=\overline{f\left(t^{-1}, \theta_{t^{-1}}(x)\right)},
\end{gathered}
$$

$\forall f, g \in C_{c}(\mathcal{G}),(t, x) \in \mathcal{G}$, and $z=d(t, x)=\theta_{t^{-1}}(x)$. Then, $C^{*}(\mathcal{G})$ is the enveloping $C^{*}$-algebra of the completion of $C_{c}(\mathcal{G})$ with respect to the norm $\|f\|_{I}=$ $\sup \left\{\|f\|_{I, r},\|f\|_{I, d}\right\}$, where $\|f\|_{I, r}=\sup _{x \in X} \int_{A_{x}^{-1}}|f(s, x)| d \lambda(s)$ and $\|f\|_{I, d}=$ $\sup _{x \in X} \int_{A_{x}^{-1}}\left|f\left(s^{-1}, \theta_{s^{-1}}(x)\right)\right| d \lambda(s)$. In fact, by [16, Proposition 1.11, p. 58] we may suppose that $C_{c}(\mathcal{G}) \subseteq C^{*}(\mathcal{G})$. The ${ }^{*}$-algebra $C_{c}(\mathcal{G})$ has another important topological structure, the one given by the locally convex inductive limit topology $\tau_{\mathcal{G}}$ induced by the direct system $\left\{C_{K}(\mathcal{G})\right\}_{K}$, where $K$ runs in the family of compact subsets of $\mathcal{G}$ and $C_{K}(\mathcal{G}):=\left\{f \in C_{c}(\mathcal{G}): \operatorname{supp}(f) \subseteq K\right\}$.

If $\mathcal{B}$ is a Fell bundle over $G$ and $K$ is a compact subset of $G$, we set $C_{K}(\mathcal{B}):=$ $\left\{f \in C_{0}(\mathcal{B}): \operatorname{supp}(f) \subseteq K\right\}$, and $C_{c}(\mathcal{B}):=\left\{f \in C_{0}(\mathcal{B}): \operatorname{supp}(f)\right.$ is compact $\}$. Recall from [9] that $C_{c}(\mathcal{B})$ is a dense ${ }^{*}$-subalgebra of $C^{*}(\mathcal{B})$. As previously with $C_{c}(\mathcal{G}), C_{c}(\mathcal{B})$ is endowed with the locally convex inductive limit topology induced by the system $\left\{C_{K}(\mathcal{B})\right\}_{K}$, where $K$ runs in the family of compact subsets of $G$.

Proposition 3.1. Let $\mathcal{G}$ be the groupoid associated with the partial action $\theta$ on $X$, and $\mathcal{B}$ the Fell bundle associated with the partial action $\sigma=\Phi(\theta)$ on $A:=C_{0}(X)$. For $f \in C_{c}(\mathcal{G})$, and $t \in G$, consider the function $f_{t}: X \rightarrow \mathbb{C}$ given by $f_{t}(x)=$ $f(t, x)$. Let $\phi: C_{c}(\mathcal{G}) \rightarrow C_{c}(\mathcal{B})$ be the map given by $\phi(f)=\hat{f}$, where $\hat{f}: G \rightarrow \mathcal{B}$ is such that $\hat{f}(t)=\Delta(t)^{-1 / 2} f_{t} \delta_{t}, \forall t \in G$. Then $\phi$ is an injective homomorphism of $*_{-}$ algebras. Moreover, if we consider on $C_{c}(\mathcal{G})$ and $C_{c}(\mathcal{B})$ the corresponding inductive limit topologies, then $\phi$ is continuous with dense range.

Proof. We leave it to the reader to check that actually $\hat{f} \in C_{c}(\mathcal{B})$. Now, it is clear that $\phi$ is linear and that $\phi(f)=0$ implies $f=0$; so $\phi$ is injective.

To see that $\phi$ preserves multiplication, consider $h=f \star g$. Then we have that $h(t, x)=\int_{A_{x}^{-1}} f(s, x) g\left(s^{-1} t, \theta_{s^{-1}}(x)\right) d \lambda(s)$, and therefore

$$
h_{t}(x)=\int_{A_{x}^{-1}} f_{s}(x) g_{s^{-1} t}\left(\theta_{s^{-1}}(x)\right) d \lambda(s)=\int_{G} \sigma_{s}\left(\sigma_{s^{-1}}\left(f_{s}\right) g_{s^{-1} t}\right)(x) d \lambda(s) .
$$

In other words, $\hat{h}(t)=\frac{1}{\sqrt{\Delta(t)}} \int_{G} \sigma_{s}\left(\sigma_{s^{-1}}\left(f_{s}\right) g_{s^{-1} t}\right)(x) d \lambda(s) \delta_{t}$. On the other hand,

$$
\hat{f} * \hat{g}(t)=\int_{G} \sigma_{s}\left(\sigma_{s^{-1}}\left(\frac{f_{s}}{\sqrt{\Delta(s)}}\right) \frac{g_{s^{-1} t}}{\sqrt{\Delta\left(s^{-1} t\right)}}\right) d \lambda(s) \delta_{t}=\int_{G} \sigma_{s}\left(\sigma_{s^{-1}}\left(f_{s}\right) g_{s^{-1} t}\right) \frac{d \lambda(s)}{\sqrt{\Delta(t)}} \delta_{t}
$$

and therefore $\hat{h}=\hat{f} * \hat{g}$. As for the involution:

$$
(\hat{f})^{*}(t)=\Delta\left(t^{-1}\right) \hat{f}\left(t^{-1}\right)^{*}=\Delta\left(t^{-1}\right) \sigma_{t}\left(\frac{1}{\sqrt{\Delta\left(t^{-1}\right)}} \overline{f_{t^{-1}}}\right) \delta_{t}=\frac{1}{\sqrt{\Delta(t)}} \overline{\sigma_{t}\left(f_{t^{-1}}\right)} \delta_{t}
$$

and

$$
\widehat{\left(f^{\star}\right)}(t)=\frac{1}{\sqrt{\Delta(t)}} f_{t}^{*} \delta_{t}=\frac{1}{\sqrt{\Delta(t)}} \overline{\sigma_{t}\left(f_{t^{-1}}\right)} \delta_{t}
$$

$\operatorname{thus}(\hat{f})^{*}=\widehat{\left(f^{\star}\right)}$. 
To see that $\phi$ is continuous, consider $\iota: C_{c}(\mathcal{G}) \rightarrow C_{c}(\mathcal{B})$ such that $\iota(f)(t)=f_{t} \delta_{t}$. Note that for every compact subset $K$ of $\mathcal{G}$ the diagram below commutes:

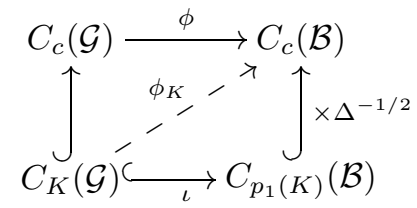

So $\phi_{K}$ is the composition of two continuous maps, and therefore it is continuous. Then $\phi$ is continuous. It remains to prove that $\phi\left(C_{c}(\mathcal{G})\right)$ is dense in $C_{c}(\mathcal{B})$ or, which is the same, that $\iota\left(C_{c}(\mathcal{G})\right)$ is dense in $C_{c}(\mathcal{B})$. Since $\iota\left(C_{c}(\mathcal{G})\right)$ is a $C(G)$-submodule of $C_{c}(\mathcal{B})$ it suffices, by [9, II-14.6], to prove that $\left\{f_{t} \delta_{t}: f \in C_{c}(\mathcal{G})\right\}$ is dense in $B_{t}$, $\forall t \in G$. That is, we must show that $\left\{f_{t}: f \in C_{c}(\mathcal{G})\right\}$ is dense in $D_{t}, \forall t \in G$. So let $a \in C_{c}\left(X_{t}\right)$. Since $\{t\} \times \operatorname{supp}(a) \subseteq \mathcal{G}$ is compact, there exists a neighborhood $V$ of $\{t\} \times \operatorname{supp}(a)$ such that $\bar{V}$ is compact and $\bar{V} \subseteq \mathcal{G}$. By Urysohn's Lemma, there exists $g \in C_{c}(\mathcal{G})$ such that $0 \leq g \leq 1, g(t, x)=1, \forall x \in\{t\} \times \operatorname{supp}(a)$, and $g(s, x)=0$ if $(s, x) \notin V$. Let us then define $f: \mathcal{G} \rightarrow \mathbb{C}$ such that $f(s, x)=0$ if $(s, x) \notin V, f(s, x)=a(x) g(s, x)$ if $(s, x) \in V$. Then $f \in C_{c}(\mathcal{G})$ and $f_{t}=a$. Thus, $\left\{f_{t}: f \in C_{c}(\mathcal{G})\right\}=C_{c}\left(X_{t}\right)$, which is dense in $D_{t}$.

Lemma 3.2. Let $K \subseteq G$ be a compact and symmetric subset of $G$. Then, for every $f \in C_{c}(\mathcal{G})$ such that $\operatorname{supp}(\phi(f)) \subseteq K$ we have that $\|f\|_{I} \leq \sqrt{\|\Delta\|_{K}}\|\phi(f)\|_{1}$. (Here $\|\Delta\|_{K}=\max _{t \in K} \Delta(t)$, and $\|\cdot\|_{1}$ is the norm on $L^{1}(\mathcal{B})$.)

Proof. We have $\|\phi(f)\|_{1}=\int_{G}\left\|\frac{1}{\sqrt{\Delta(t)}} f_{t}\right\| d \lambda(t) \geq \frac{1}{\sqrt{\|\Delta\|_{K}}} \int_{K}\left\|f_{t}\right\| d \lambda(t)$. Therefore, $\|\hat{f}\|_{1} \geq \frac{1}{\sqrt{\|\Delta\|_{K}}}\|f\|_{I, r}$. Since $\|\hat{f}\|_{1}=\left\|(\hat{f})^{*}\right\|_{1}=\left\|\widehat{\left(f^{\star}\right)}\right\|_{1}$ and $\|f\|_{I, r}=\left\|f^{\star}\right\|_{I, d}$, we have $\|\hat{f}\|_{1} \geq \frac{1}{\sqrt{\|\Delta\|_{K^{-1}}}}\|f\|_{I, d}$. Now the result follows from the symmetry of $K$.

Theorem 3.3. Let $R_{\mathcal{G}}$ be the family of nondegenerate $\left(\tau_{\mathcal{G}}, W O T\right)$-continuous representations of $C_{c}(\mathcal{G})$, and $R$ the family of nondegenerate $(\tau, W O T)$-continuous representations of $C_{c}(\mathcal{B})$, where $\tau$ is the inductive limit topology on $C_{c}(\mathcal{B})$. Then

1. the correspondence $R \rightarrow R_{\mathcal{G}}$ given by $\pi \longmapsto \pi \circ \phi$ is a bijection;

2. $\phi$ extends uniquely to an isomorphism $C^{*}(\mathcal{G}) \rightarrow C^{*}(\mathcal{B})$ of $C^{*}$-algebras.

Proof. It is clear from Proposition 3.1 that the correspondence under our attention is injective. Suppose now that $\tilde{\pi} \in R_{\mathcal{G}}, \tilde{\pi}: C_{c}(\mathcal{G}) \rightarrow B(H)$, and let $\pi$ : $\phi\left(C_{c}(\mathcal{G})\right) \rightarrow B(H)$ be defined by $\pi\left(\phi\left(f^{\prime}\right)\right)=\tilde{\pi}\left(f^{\prime}\right)$. Notice that $\phi\left(C_{c}(\mathcal{G})\right) \cap$ $C_{K}(\mathcal{B})$ is \|\|$_{\infty}$-dense in $C_{K}(\mathcal{B})$. Therefore, if $f=\phi\left(f^{\prime}\right), f^{\prime} \in C_{c}(\mathcal{G})$, then $\|\pi(f)\|=\left\|\pi\left(\phi\left(f^{\prime}\right)\right)\right\|=\left\|\tilde{\pi}\left(f^{\prime}\right)\right\| \leq\left\|f^{\prime}\right\|_{I}$, where the last inequality is due to [16. II-1.22]. Now, by Lemma 3.2 we have $\left\|f^{\prime}\right\|_{I} \leq \sqrt{\|\Delta\|_{K}}\left\|\phi\left(f^{\prime}\right)\right\|_{1}=\sqrt{\|\Delta\|_{K}}\|f\|_{1} \leq$ $\sqrt{\|\Delta\|_{K}} \lambda(K)\|f\|_{\infty}$. Then the restriction of $\pi$ to $C_{K}(\mathcal{B}) \cap \phi\left(C_{c}(\mathcal{G})\right)$ extends uniquely to a bounded operator $\pi_{K}$ on $C_{K}(\mathcal{B})$. It is clear that if $g \in C_{K}(\mathcal{B})$ and $g \in C_{K^{\prime}}(\mathcal{B})$, then $\pi_{K}(g)=\pi_{K^{\prime}}(g)$. Thus, we obtain a $\tau$-continuous linear map $\pi: C_{c}(\mathcal{B}) \rightarrow$ $B(H)$ that preserves the involution. Let us see that $\pi$ also preserves the product. Let $f, g \in C_{c}(\mathcal{B})$. Then there exists a compact set $K \subseteq G$ such that $f, g \in C_{K}(\mathcal{B})$. Given $\epsilon>0$, there exist $f^{\prime}, g^{\prime} \in C_{c}(\mathcal{G})$, with $p_{1}\left(\operatorname{supp}\left(f^{\prime}\right)\right), p_{1}\left(\operatorname{supp}\left(g^{\prime}\right)\right) \subseteq K$, such that $\left\|f-\phi\left(f^{\prime}\right)\right\|_{\infty}+\left\|g-\phi\left(g^{\prime}\right)\right\|_{\infty}<\epsilon$. Note that, if $\tilde{f}, \tilde{g} \in C_{K}(\mathcal{B})$, then 
$\tilde{f} * \tilde{g} \in C_{K^{2}}(\mathcal{B})$. Then we have

$$
\begin{aligned}
\|\pi(f * g)-\pi(f) \pi(g)\|=\| \pi(f * g)-\tilde{\pi}\left(f^{\prime} \star g^{\prime}\right) & +\tilde{\pi}\left(f^{\prime}\right) \tilde{\pi}\left(g^{\prime}\right)-\pi(f) \pi(g) \| \\
\leq\left\|\pi(f * g)-\pi\left(\phi\left(f^{\prime}\right)\right) \pi\left(\phi\left(g^{\prime}\right)\right)\right\| & \\
& +\left\|\pi\left(\phi\left(f^{\prime}\right)\right) \pi\left(\phi\left(g^{\prime}\right)\right)-\pi(f) \pi(g)\right\| \\
\leq \sqrt{\|\Delta\|_{K^{2}}} \lambda\left(K^{2}\right) \| f * g & -\phi\left(f^{\prime}\right) * \phi\left(g^{\prime}\right) \|_{\infty} \\
& +\left\|\pi\left(\phi\left(f^{\prime}\right)\right) \pi\left(\phi\left(g^{\prime}\right)\right)-\pi(f) \pi(g)\right\| .
\end{aligned}
$$

Now

$$
\begin{aligned}
\left\|\pi\left(\phi\left(f^{\prime}\right)\right) \pi\left(\phi\left(g^{\prime}\right)\right)-\pi(f) \pi(g)\right\| & =\left\|\pi\left(\phi\left(f^{\prime}\right)\right) \pi\left(\phi\left(g^{\prime}\right)-g\right)+\pi\left(\phi\left(f^{\prime}\right)-f\right) \pi(g)\right\| \\
& \leq\left\|\pi\left(\phi\left(f^{\prime}\right)\right)\right\| \sqrt{\|\Delta\|_{K}} \lambda(K)\left\|\phi\left(g^{\prime}\right)-g\right\|_{\infty} \\
& \quad+\|\pi(g)\| \sqrt{\|\Delta\|_{K}} \lambda(K)\left\|\phi\left(f^{\prime}\right)-f\right\|_{\infty} \\
& \leq M\|\Delta\|_{K} \lambda(K)^{2} \epsilon
\end{aligned}
$$

where $M=\max \left\{\|f\|_{\infty}+\epsilon,\|g\|_{\infty}+\epsilon\right\}$. On the other hand,

$$
\begin{aligned}
\left\|f * g-\phi\left(f^{\prime}\right) * \phi\left(g^{\prime}\right)\right\|_{\infty} & =\left\|f *\left(g-\phi\left(g^{\prime}\right)\right)+\left(f-\phi\left(f^{\prime}\right)\right) * \phi\left(g^{\prime}\right)\right\|_{\infty} \\
& \leq \epsilon\left(\int_{K}\|f(s)\| d s+\int_{K}\left\|\phi\left(g^{\prime}\right)(s)\right\| d s\right) \\
& \leq 2 M \lambda(K) \epsilon .
\end{aligned}
$$

Consequently,

$$
\|\pi(f * g)-\pi(f) \pi(g)\| \leq M \lambda(K)\left(2 \sqrt{\|\Delta\|_{K^{2}}} \lambda\left(K^{2}\right)+\|\Delta\|_{K} \lambda(K)\right) \epsilon, \forall \epsilon>0 .
$$

Hence $\pi(f * g)=\pi(f) \pi(g)$. Now, by the definition of $\pi$, we have that $\tilde{\pi}=\pi \circ \phi$.

For proving 2, note that $C^{*}(\mathcal{G})$ is the completion of $C_{c}(\mathcal{G})$ with respect to the norm $\|\cdot\|: C_{c}(\mathcal{G}) \rightarrow \mathbb{R}$ such that $\left\|f^{\prime}\right\|=\sup \left\{\left\|\pi\left(f^{\prime}\right)\right\|: \pi \in R_{\mathcal{G}}\right\}$; on the other hand, by [9, VIII-13.8], we have that $C^{*}(\mathcal{B})$ is the completion of $\phi\left(C_{c}(\mathcal{G})\right)$ relative to the norm given by $\|f\|=\sup \{\|\pi(f)\|: \pi \in R\}$. Thus $\left\|f^{\prime}\right\|=\left\|\phi\left(f^{\prime}\right)\right\|, \forall f^{\prime} \in C_{c}(\mathcal{G})$, and then $\phi$ extends by continuity to an isomorphism of $C^{*}(\mathcal{G})$ onto $C^{*}(\mathcal{B})$.

\section{REFERENCES}

[1] F. Abadie, Enveloping actions and Takai duality for partial actions, J. Funct. Anal. 197 (2003), 14-67.

[2] R. Exel, Circle actions on $C^{*}$-algebras, partial automorphisms and a generalized PimsnerVoiculescu exact sequence, J. Funct. Anal. 122 (1994), 361-401. MR 95g:46122

[3] R. Exel, The Bunce-Deddens algebras as crossed products by partial automorphisms, Bol. Soc. Brasil. Mat. 25 (2) (1994), 173-179. MR 95m:46091

[4] R. Exel, Approximately finite $C^{*}$-algebras and partial automorphisms, Math. Scand. 77 (1) (1995), 281-288. MR 97e:46085

[5] R. Exel, Twisted partial actions, a classification of regular $C^{*}$-algebraic bundles, Proc. London Math. Soc. 74 (1997), 417-443. MR 98d:46075

[6] R. Exel, Partial actions of groups and actions of inverse semigroups, Proc. Amer. Math. Soc. 126 (1998), no. 12, 3481-3494. MR 99b:46102

[7] R. Exel and M. Laca, Cuntz-Krieger algebras for infinite matrices, J. Reine Angew. Math. 512 (1999), 119-172. MR 2000i:46064

[8] R. Exel, M. Laca, and J. Quigg, Partial dynamical systems and $C^{*}$-algebras generated by partial isometries, J. Operator Theory 47 (2002), no. 1, 169-186. MR 2003f:46108 
[9] J. M. Fell and R. S. Doran, Representations of *-algebras, locally compact groups, and Banach *-algebraic bundles, Pure and Applied Mathematics 125 and 126, Academic Press, Boston, MA, 1988. MR 90c:46001, MR 90c:46002

[10] A. Haefliger, Structures feuilletées et cohomologie à valeur dans un faiseau de groupoïdes, Comment. Math. Helv. 32 (1958), 248-329. MR 20:6702

[11] K. Maclanahan, K-theory for partial crossed products by discrete groups, J. Funct. Anal. 130 (1995) 77-117. MR 96i:46083

[12] A. Nica, On a groupoid construction for actions of certain inverse semigroups, Internat. J. Math. 5 (1994), 349-372. MR 95h:46086

[13] A. L. T. Paterson, Groupoids, inverse semigroups, and their operator algebras, Progress in Mathematics 170, Birkhaüser Boston, 1999. MR 2001a:22003

[14] J. C. Quigg and I. Raeburn, Characterizations of crossed products by partial actions, J. Operator Theory 37 (1997), No. 2, 311-340. MR 99a:46121]

[15] J. C. Quigg and N. Sieben, $C^{*}$-actions of $r$-discrete groupoids and inverse semigroups, J. Austral. Math. Soc. Ser. A 66 (1999), 143-167. MR 2000k:46097]

[16] Jean Renault, A groupoid approach to $C^{*}$-algebras, Lecture Notes in Math. 793, SpringerVerlag, Berlin, 1980. MR 82h:46075

[17] N. Sieben, $C^{*}$-crossed products by partial actions and actions of inverse semigroups, J. Austral. Math. Soc. Ser. A 63 (1997), 32-46. MR 2000b:46124

Centro de Matemática, Facultad de Ciencias, Universidad de la República, Iguá 4225, 11400, Montevideo, Uruguay

E-mail address: fabadie@cmat.edu.uy 